\title{
Best $L_{p}$ Approximation*
}

\author{
By S. W. Kahng
}

\begin{abstract}
A new algorithm is presented for the best $L_{p}$ approximation of a continuous function over a discrete set or a finite interval with $2<p<\infty$. Methods to accelerate the convergence of the Rice-Usow extension of Lawson's algorithm as well as the new algorithm are presented, and the result of a numerical example is given.
\end{abstract}

1. Introduction. In 1961 Lawson [1] gave an algorithm for finding the Chebyshev approximation on a finite point set. In 1968 Rice and Usow [2] extended the algorithm to the best $L_{p}$ approximation with $2<p<\infty$. These algorithms apply on a finite point set but not on a finite interval, and their convergences are slow.

In this paper, we describe a new algorithm for the best $L_{p}$ approximation. It is an adaptation of Newton-Raphson's method, and is applicable both on a finite interval and on a finite point set with $2<p<\infty$. The convergence of this iterative method is quadratic. We also give a scheme for accelerating the convergence of the extended Lawson algorithm as well as the new method. Finally, the result of a numerical example is given.

2. Best $L_{p}$ Approximation. Let $f(x)$ be continuous in $[0,1]$; let $\left\{\varphi_{i}(x)\right\}, i=$ $1,2, \cdots, n$, be a set of continuous and linearly independent functions in $[0,1]$; let $L(A, x)=\sum_{i=1}^{n} a_{i} \varphi_{i}(x)$, where $A=\left(a_{1}, a_{2}, \cdots, a_{n}\right)^{T}$; let $2<p<\infty$; and let $w(x)$ be a nonnegative and Riemann integrable function in $[0,1]$. In this paper, we say $L(A, x)$ is the best $L_{p, w}$ (or $\left.L_{p}\right)$ approximation of $f(x)$ in $[0,1]$, if

$$
\int_{0}^{1}|f(x)-L(A, x)|^{p} w(x) d x \leqq \int_{0}^{1}|f(x)-L(B, x)|^{p} w(x) d x
$$

for all $B=\left(b_{1}, b_{2}, \cdots, b_{n}\right)^{T}$.

The new algorithm for the best $L_{p}$ approximation of

$$
F(A)=\int_{0}^{1}|f(x)-L(A, x)|^{p} d x
$$

is as follows:

Starting from the initial coefficient set $A_{0}$, at the $k$ th iteration

Step 1. Set $w_{k}(x)=\left|f(x)-L\left(A_{k-1}, x\right)\right|^{p-2}$.

Step 2. Find the least squares approximation $L\left(B_{k}, x\right)$ of the function $f(x)$ with the weight function $w_{k}(x)$.

Step 3. Set $A_{k}=\left\{(p-2) A_{k-1}+B_{k}\right\} /(p-1)$.

Received November 20, 1970, revised August 24, 1971.

AMS 1969 subject classifications. Primary 4115, 6520.

Key words and phrases. Polynomial approximation, Newton-Raphson method.

* This work was supported by the Department of the Navy under Contract N00017-62-C-0604.

Presented at the 1971 SIAM National Meeting.

Copyright @ 1972, American Mathematical Society 
We show below that the above algorithm is a rearranged form of Newton-Raphson's method. In view of the fact that (1) is a strictly convex function of $A$ [3], it follows that the iteration always converges [4].

The minimization of (1) may be achieved by finding the roots of

$$
\partial F(A) / \partial a_{i}=0, \quad i=1,2, \cdots, n,
$$

whereas (2) can be solved iteratively by finding the solutions of

$$
\frac{\partial F(A)}{\partial a_{i}}+\sum_{i=1}^{n} \frac{\partial^{2} F(A)}{\partial a_{i} \partial a_{j}} \Delta a_{i}=0
$$

and replacing $a_{i}$ with $a_{i}+\Delta a_{i}$ at each iteration, which is the Newton-Raphson method.

From (1) we find, at the $k$ th iteration, that

$$
\begin{aligned}
\frac{\partial F\left(A_{k-1}\right)}{\partial a_{i}} & =-p \int_{0}^{1}\left|f(x)-L\left(A_{k-1}, x\right)\right|^{p-1} \operatorname{sgn}\left(\varphi_{i}(x), f(x)-L\left(A_{k-1}, x\right)\right) d x \\
& =-p \int_{0}^{1} w_{k}(x)\left(f(x)-L\left(A_{k-1}, x\right)\right) \varphi_{i}(x) d x
\end{aligned}
$$

and

$$
\frac{\partial^{2} F\left(A_{k-1}\right)}{\partial a_{j} \partial a_{i}}=p(p-1) \int_{0}^{1} w_{k}(x) \varphi_{i}(x) \varphi_{j}(x) d x
$$

where

$$
\begin{aligned}
\operatorname{sgn}(x, y)=x & \text { if } y>0 \\
=-x & \text { if } y<0 \\
=0 & \text { if } y=0
\end{aligned}
$$

and

$$
w_{k}(x)=\left|f(x)-L\left(A_{k-1}, x\right)\right|^{p-2} .
$$

Substituting (4) and (5) into (3), we have

$$
(p-1) H_{k} \Delta A_{k}=D_{k}-H_{k} A_{k-1},
$$

where $H_{k}$ is an $n$-by- $n$ matrix whose $i, j$ th entry is

$$
\int_{0}^{1} w_{k}(x) \varphi_{i}(x) \varphi_{j}(x) d x
$$

where $\Delta A_{k}=\left(\Delta a_{1}, \Delta a_{2}, \cdots, \Delta a_{n}\right)^{T}$, and where

$$
D_{k}=\left(\int_{0}^{1} w_{k}(x) f(x) \varphi_{1}(x) d x, \int w_{k}(x) f(x) \varphi_{2}(x) d x, \cdots, \int_{0}^{1} w_{k}(x) f(x) \varphi_{n}(x) d x\right)^{T} .
$$

By noting that $L\left(B_{k}, x\right)$ is the $L_{2, w k}$ approximation of $f(x)$, we have $H_{k} B_{k}=D_{k}$. Therefore,

$$
(p-1) H_{k} \Delta A_{k}=H_{k}\left(B_{k}-A_{k-1}\right)
$$


and

$$
\Delta A_{k}=\frac{1}{p-1}\left(B_{k}-A_{k-1}\right)
$$

or

$$
A_{k}=A_{k-1}+\Delta A_{k}=\frac{1}{p-1}\left\{(q-2) A_{k-1}+B_{k}\right\}
$$

and the above algorithm is a rearranged form of Newton-Raphson's method.

It is noted that the matrix $H_{k}$ is nonsingular [5], which implies that the convergence of the iteration is quadratic [4] and that the replacement of the operator "integration from 0 to 1" by the operator "summation over a discrete. set" does not affect the validity of the algorithm.

3. Acceleration of the Convergence. Convergence of the new method is slow when the starting point $A_{0}$ is not sufficiently close to the best approximation, say $A^{*}$. As $p$ is increased, more iterations are needed to achieve results of the same accuracy. However, the following acceleration scheme was found to be effective and, in all test cases, the number of iterations required was reduced to one third.

At the $k$ th iteration, use $p_{k}$ in place of $p$ in the algorithm where

$$
p_{1}=2, \quad p_{k}=\min \left(p, 2 p_{k-1}\right) \text {. }
$$

We made an analogous modification to the Rice-Usow extention of Lawson's algorithm (LRU algorithm) by replacing $p$ with $p_{k}$ at the $k$ th iteration and found it to be equally effective. These modified algorithms converge, since $p_{k}$ becomes $p$ after several iterations.

The new algorithm and LRU method, along with their acceleration schemes, were applied to an example where summation over the equally spaced points was the operator. The results are shown in Table 1.

TABLE 1. Number of iterations required to approximate $\exp (10 x)$, in $[0,1]$ at equally spaced points, by a polynomial of degree 5, to 4-figure accuracy.

\begin{tabular}{rcccc}
\hline$p \cdot$ & No. of Points & New Method & LRU Method** & $\begin{array}{c}\text { LRU Method With } \\
\text { Modification }\end{array}$ \\
\hline 50 & 30 & 8 & 24 & 8 \\
50 & 30 & $26^{*}$ & & 9 \\
50 & 50 & 14 & 28 & 10 \\
100 & 50 & 20 & 31 & \\
\hline
\end{tabular}

* No acceleration scheme was used.

** Rice-Usow extension of Lawson's method.

4. Conclusion. The new algorithm enjoys several advantages over the LRU algorithm. $\left\{\varphi_{i}\right\}$ does not have to be a Chebyshev set, and the algorithm is applicable to the approximation problem for integrals. The iteration does not have to be restarted, and one will not "accidentally" set the weight function to zero at any point. 
Consequently, the new algorithm is simpler to program and has wider applications. With the proposed acceleration scheme the convergence seems to be faster. However, the LRU method, with the acceleration scheme proposed in this paper, showed even faster convergence.

Applied Physics Laboratory

Johns Hopkins University

Silver Spring, Maryland 20910

1. C. L. Lawson, Contribution to the Theory of Linear Least Maximum Approximations, Ph.D. Thesis, University of California, Los Angeles, Calif., 1961, pp. 55-61.

2. J. R. RICE \& K. H. Usow, "The Lawson algorithm and extensions," Math. Comp., v. 22, 1968, pp. 118-127. MR 38 \#463.

3. G. H. Hardy, J. E. Littlewood \& G. Pólya, Inequalities, Cambridge Univ. Press, New York, 1934, p. 146.

4. J. M. ORTEGA \& W. C. RHEInboldt, Iterative Solution of Nonlinear Equations in Several Variables, Academic Press, New York, 1970, pp. 501-506.

5. J. R. RICE, The Approximation of Functions. Vol. I: Linear Theory, Addison-Wesley, Reading, Mass., 1964, p. 32. MR 29 \# 3795. 